Pinto, MLM, Queiroz, MP, Santos, ABMV, Silva, NR, Pereira, MTL \& Campos, RS. (2020). Teenage pregnancy: dietary patterns and correlation with their socioeconomic profile. Research, Society and Development, 9(7):1-21. e169973976.

\title{
Gestação na adolescência: padrões alimentares e correlação com seu perfil socioeconômico
}

Teenage pregnancy: dietary patterns and correlation with their socioeconomic profile Embarazo adolescente: patrones dietéticos y correlación con su perfil socioeconómico

Recebido: 26/04/2020 | Revisado: 27/04/2020 | Aceito: 30/04/2020 | Publicado: 05/05/2020

\section{Marina Lins Mendes Pinto}

ORCID: https://orcid.org/0000-0001-6779-4042 Universidade Federal de Campina Grande, Brasil E-mail: marilinsmendes@gmail.com.br Michelly Pires Queiroz ORCID: https://orcid.org/0000-0002-6371-1833 Universidade Federal de Campina Grande, Brasil E-mail: queiroz_m.p@hotmail.com Ana Beatriz Macêdo Venâncio dos Santos

ORCID: https://orcid.org/0000-0002-1061-6496 Universidade Federal de Campina Grande, Brasil E-mail: biabeatrizvenancio@gmail.com Naryelle da Rocha Silva ORCID: https://orcid.org/0000-002-1924-0106 Universidade Federal de Pernambuco, Brasil E-mail: naryelle_rocha@hotmail.com Maria Tereza Lucena Pereira ORCID: https://orcid.org/0000-0002-5857-9268 Universidade Federal de Campina Grande, Brasil

E-mail: mariatereza-lucena@hotmail.com Raíra da Silva Campos

ORCID: https://orcid.org/0000-0001-9653-8056 Universidade Federal de Campina Grande, Brasil E-mail: raira965@hotmail.com 


\title{
Resumo
}

A gravidez na adolescência vem sendo considerada como um problema de saúde pública, podendo gerar complicações, como: síndromes hipertensivas gestacionais, diabetes mellitus gestacional, mortalidade infantil, dentre outras. Parte desse pressuposto a necessidade de avaliar o padrão alimentar dessa população, através de métodos de inquéritos alimentares, que devem abranger e compreender diferentes dimensões da vida da gestante. Este estudo tem como objetivo analisar o consumo alimentar de gestantes adolescentes atendidas em Unidades Básicas de Saúde, correlacionando com seu estado nutricional e fator socioeconômico. Tratase de uma pesquisa quantitativa, descritiva e transversal, ocorreu no município de Cuité/PB, com 15 gestantes adolescentes assistidas por Unidades Básicas de Saúde. Aplicou-se um questionário, o qual compreendia os seguintes dados: Informações gerais, dados obstétricos, avaliação antropométrica, dados socioeconômicos e um Questionário de Frequência Alimentar. A partir deste, observou-se a prevalência de baixa renda, onde a maioria das entrevistadas recebem benefícios sociais do governo, e grande parte encontrava-se com sobrepeso e obesidade. No que condiz à escolha alimentar, percebeu-se a preferência por alimentos processados e ultraprocessados, e uma baixa frequência os alimentos in natura e minimamente processados. Ao relacionar estes dados com o perfil socioeconômico, constatou-se que os fatores econômicos tendem a influenciar de forma negativa o Estado Nutricional e o consumo alimentar de jovens grávidas. Os resultados apontam a necessidade de implementação de estratégias nutricionais para essa população, com enfoque nas principais dificuldades citadas anteriormente.

Palavras-chave: Consumo de alimentos; Gravidez na adolescência; Renda familiar.

\begin{abstract}
Teenage pregnancy has been considered a public health problem, and can generate complications, such as: gestational hypertensive syndromes, gestational diabetes mellitus, infant mortality, among others. From this assumption, the need to evaluate the dietary pattern of this population, through methods of food surveys, which should cover and understand different dimensions of the life of the pregnant woman. This study aims to analyze the food intake of pregnant adolescents attended in Basic Health Units, correlating with their nutritional status and socioeconomic factor. This is a quantitative, descriptive and crosssectional study, which took place in the city of Cuité/PB, with 15 adolescent pregnant women assisted by Basic Health Units. A questionnaire was applied, which comprised the following data: General information, obstetric data, anthropometric evaluation, socioeconomic data and
\end{abstract}


a Food Frequency Questionnaire. From this questionnaire, it was possible to observe the prevalence of low income, where most of the interviewees receive social benefits from the government, and most were overweight and obese. Regarding the food choice, the preference for processed and ultra-processed foods was perceived, and a low frequency of fresh and minimally processed foods. By relating these data to the socioeconomic profile, it was found that economic factors tend to negatively influence the Nutritional Status and food intake of young pregnant women. The results indicate the need to implement nutritional strategies for this population, focusing on the main difficulties mentioned above.

Keywords: Food consuption; Pregnancy in adolescence; Income.

\section{Resumen}

El embarazo adolescente se ha considerado un problema de salud pública y puede generar complicaciones, tales como: síndromes hipertensivos gestacionales, diabetes mellitus gestacional, mortalidad infantil, entre otros. Tenemos, entonces, la necesidad de evaluar el patrón dietético de esta población, a través de métodos de encuestas alimentarias, que deben abarcar y comprender diferentes dimensiones de la vida de la mujer embarazada. Este estudio tiene como objetivo analizar la ingesta de alimentos de las adolescentes embarazadas atendidas en Unidades Básicas de Salud, correlacionando con su estado nutricional y factor socioeconómico. Se trata de un estudio cuantitativo, descriptivo y transversal, que tuvo lugar en la ciudad de Cuité/PB, con 15 adolescentes embarazadas. Se aplicó un cuestionario con los siguientes datos: Información general, datos obstétricos, socioeconómicos, evaluación antropométrica y un Cuestionario de Frecuencia Alimentaria. A partir de esto, observamos la prevalencia de bajos ingresos, donde la mayoría de los entrevistados reciben beneficios del gobierno, y la mayoría tenían sobrepeso y obesidad. En cuanto a la elección de alimentos, se percibió la preferencia por los alimentos procesados y ultraprocesados, y una baja frecuencia de alimentos frescos y mínimamente procesados. Al relacionar estos datos con el perfil socioeconómico, se encontró que los factores económicos tienden a influir negativamente en el estado nutricional y la ingesta de alimentos. Los resultados indican la necesidad de implementar estrategias nutricionales, centrándose en las principales dificultades mencionadas anteriormente.

Palabras clave: Consumo de alimentos; Embarazo em adolescencia; Renta. 


\section{Introdução}

O período da adolescência é marcado por incontáveis descobertas e rápidas transformações, sejam elas biológicas e psicossociais. Tais mudanças repercutem direta e indiretamente nas escolhas alimentares, bem como na visão de imagem corporal das jovens (Oliboni \& Alvarenga, 2015). É nessa fase que ocorre o aumento do crescimento, ocasionando uma alta demanda energética no intuito de suprir essas necessidades, além de ser um momento em que o organismo se prepara para atingir a maturação sexual e a capacidade de reprodução (Kempfer et al, 2012).

A incidência da gravidez na adolescência vem sendo cada vez mais comum, sendo considerada um problema de saúde pública que pode gerar complicações decorrentes de uma gestação precoce, como por exemplo síndromes hipertensivas gestacionais, diabetes mellitus estacional, mortalidade infantil, dentre outras (Albuquerque et al, 2017).

Dentre as fases da vida de uma mulher, a gestação, assim como a adolescência, é um período que exige maior demanda energética, tornando-se uma etapa demasiadamente susceptível às inadequações nutricionais. A importância de se ter uma alimentação adequada é primordial, tendo em vista que a qualidade da alimentação pode vir a afetar o crescimento e desenvolvimento fetal, além de dificultar o perfeito desenvolvimento da gestação, devido ao fato de que a única fonte de nutrientes do concepto é derivada das reservas nutricionais da mãe (Gomes et al, 2019).

Entretanto, Tavares et al. (2014), citam em seu estudo que as escolhas alimentares do adolescente no Brasil, em geral, não são consideradas saudáveis em razão dos seus hábitos alimentares, é notória a grande ingestão de alimentos processados e ultra processados, refeições com alto valor energético, ricas em gorduras, açúcares e sódio e pobres em micronutrientes, muitas vezes adotando dietas monótonas e modismos alimentares, enquadrando as adolescentes no grupo de riscos nutricionais.

Parte desse pressuposto a necessidade de se avaliar o padrão alimentar de jovens gestantes, e, para isso, existem métodos de inquéritos alimentares, os quais devem abranger e compreender as diferentes dimensões da vida da entrevistada, como por exemplo, cultura, religião, fatores socioeconômicos e experiências individuais. Portanto, conhecer o consumo alimentar é uma opção de grande valia para relacionar a alimentação com a saúde e/ou a doença (Silva \&Vasconcelos, 2012).

O interesse do conhecimento acerca dos padrões alimentares de gestantes adolescentes baseia-se na perspectiva de uma maior presença de intervenções nutricionais com prioridade 
nos déficits que a população estudada apresenta e criações de novas políticas públicas, caso seja necessário, garantindo, assim, uma assistência nutricional no pré-natal eficaz. Sendo assim, o presente estudo tem como objetivo analisar o consumo alimentar de gestantes adolescentes atendidas em Unidades Básicas de Saúde (UBS) do município de Cuité/PB, correlacionando com o estado nutricional e fator socioeconômico das mesmas.

\section{Metodologia}

Trata-se de um estudo do tipo descritivo com abordagem transversal e quantitativa, que segundo Pereira (2018), visa analisar matematicamente os dados colhidos, formando uma base para as conclusões da pesquisa.

Participaram da pesquisa 15 mulheres grávidas que se encaixavam no perfil de adolescentes de acordo com a OMS (Brasil, 2013), isto é, entre 12 e 19 anos de idade, desde que estivessem sendo acompanhadas pelas Unidades Básicas de Saúde do município. Foram excluídas da pesquisa gestantes maiores de 19 anos, aquelas que não estavam sendo atendidas pela Assistência Pré-Natal das UBS eleitas, assim como aquelas que não assinaram o Termo de Consentimento Livre e Esclarecido, negando sua participação. Imprevistos como adolescentes que no dia da visita tivessem chegado ao fim de sua gestação (parto ou aborto), ausência da entrevistada na sua residência por 3 visitas consecutivas e morar em zona rural, também foram considerados critérios de exclusão.

Através do prontuário solicitado em cada UBS foi possível coletar o quantitativo de gestantes adolescentes que a área cobria, assim como os seguintes dados: endereço para a realização de visita domiciliar, Data da Última Menstruação (DUM), Data Provável do Parto (DPP) e Idade Gestacional (IG).

Para avaliar o estado nutricional foi aferido o Índice de Massa Corporal (IMC), o qual foi classificado baseado nas curvas de IMC por idade gestacional proposta por Atalah (1997). Aferiu-se o peso corporal para a avaliação do IMC com o subsídio de uma balança antropométrica de plataforma e eletrônica devidamente calibrada. A altura foi medida através de uma fita métrica posta em uma parede de superfície plana e lisa. As gestantes foram orientadas a se posicionarem descalças, com calcanhares juntos, costas eretas e braços estendidos ao lado do corpo, no intuito de evitar oscilações no peso e estatura. O prontuário de cada participante foi consultado para verificar o peso pré-gravídico e realizar imediatamente a avaliação do IMC pré-gestacional, sendo feito o cálculo da Idade Gestacional daquelas gestantes que não tinham essa informação no prontuário. 
No que tange os dados socioeconômicos, foi aplicado um questionário semiestruturado em formato de entrevista, contendo informações como: estado civil, escolaridade, renda média familiar, ocupação, densidade de morador por cômodo, recebimento de benefício de transferência de renda, dentre outras, por pesquisadoras anteriormente treinadas. Os nomes das entrevistadas foram preservados, sendo identificadas apenas por numerações de questionário no intuito de evitar desconfortos e constrangimentos no decorrer da conversa.

Com relação à renda familiar, as participantes foram agrupadas em 8 grupos, tais quais: Grupo 1: até 1/4 do salário mínimo; Grupo 2: mais de 1/4 a 1/2 do salário mínimo; Grupo 3: mais de 1/2 até 1 salário mínimo; Grupo 4: mais de 1 até 2 salários mínimos; Grupo 5: mais de 2 até 3 salários mínimos; Grupo 6: mais de 3 até 5 salários mínimos; Grupo 7: mais de 5 salários mínimos e Grupo 8: sem rendimento. Já a densidade de moradores por cômodo, foram separadas em 4 (quatro) grupos: Grupo 1: até 0,5 morador por cômodo; Grupo 2: mais de 0,5 a 1 morador por cômodo; Grupo 3: mais de 1 a 2 moradores por cômodo; Grupo 4: mais de 2 moradores por cômodo.

Quanto ao grau de escolaridade, as gestantes adolescentes foram agrupadas da seguinte forma: Grupo 1: ensino fundamental incompleto; Grupo 2: ensino fundamental completo; Grupo 3: ensino médio incompleto e Grupo 4: ensino médio completo.

Para a análise de consumo alimentar das gestantes adolescentes, foi aplicado um Questionário de Frequência Alimentar (QFA) validado e adaptado à realidade do local da pesquisa.

A análise dos dados foi realizada pelo programa estatístico Statistical Package for Social Sciences (SPSS) for Windows versão 22.0. Para as análises estatísticas dos dados, foram determinadas as frequências simples e cruzamentos de variáveis.

A pesquisa atendeu as Normas Regulamentares de Pesquisa envolvendo humanos, preconizada pela Resolução $N^{\circ}$ 466/12, do Conselho Nacional de Saúde, sendo aceita pelo Comitê de Ética e Pesquisa com Seres Humanos, através do parecer de número CAAE: 86904518.4.0000.5182.

\section{Resultados e Discussão}

Ao avaliar o período gestacional, 13,3\% das participantes estavam vivenciando o primeiro trimestre gestacional, $46,7 \%$ no segundo trimestre e $40 \%$ no terceiro trimestre. 
Percebe-se uma maior concentração das participantes avaliadas vivenciando $\mathrm{s}$ dois últimos trimestres, e é justamente nessas fases da gravidez que a mãe sofre influência direta do ambiente em que está inserida, como, por exemplo, ingestão alimentar, ganho de peso e estilo de vida. Além disso, o segundo e o terceiro trimestre são determinantes para o crescimento e desenvolvimento do feto, sendo necessária uma atenção redobrada no que diz respeito à alimentação (Vitolo, 2008).

Com relação ao estado civil, mais da metade, isto é, $60 \%$ das adolescentes relataram estar solteira enquanto 33,3\% declararam uma união estável e apenas 6,7\% estava casada. De acordo com Francisquini (2010), a falta de apoio do pai da criança diante da descoberta da gestação, caracterizando uma situação conjugal inconstante, pode ser mais um fator de risco.

Além do fato de que a ausência de um companheiro tende a comprometer de alguma forma a economia da família, pois em muitos casos, essas jovens precisam recorrer financeiramente aos seus pais para ajudar com os custos, ou ainda tendo que procurar algum emprego, comprometendo todo um futuro nos estudos e sua situação econômica (Ferreira\&Ribeiro, 2010).

Tratando-se da paridade, apenas $6,7 \%$ são multigestas e $93,3 \%$ são primigestas. Ferreira e Benício (2015) informam que gestantes multigestas tendem a possuir um estado nutricional de sobrepeso e/ou obesidade.

A reincidência da gravidez na adolescência torna mais difícil a reinserção da jovem na escola, gerando uma cascata de consequências, dentre elas o perfil socioeconômico comprometido (Silva et al., 2011). Tais dados fortalecem a importância da presença assídua da gestante na assistência pré-natal, para obtenção de informações pertinentes às possíveis dificuldades vivenciadas.

Na Tabela 1, é possível observar os dados de condições socioeconômicas das gestantes adolescentes. 
Tabela 1. Distribuição das condições socioeconômicas das gestantes adolescentes do município de Cuité/PB, 2018.

\begin{tabular}{lll}
\hline \multicolumn{1}{c}{ CONDIÇÃO } & $\mathbf{N = 1 5}$ & \% \\
\hline \multicolumn{1}{c}{ Renda Média Familiar } & & \\
\hline$>1 / 2$ até 1 & 12 & $20 \%$ \\
$>1$ até 2 & 3 & $80 \%$ \\
\hline \multicolumn{1}{c}{ Benefício de Transferência de Renda } & $20 \%$ \\
\hline Recebem & 12 & \\
Não Recebem & 3 & $80 \%$ \\
\hline Densidade de morador/cômodo & 12 & $20 \%$ \\
\hline$>0,5$ até 1 & 3 & \\
\hline 1 até 2 & & \\
\hline
\end{tabular}

\section{Escolaridade}

\begin{tabular}{lll}
\hline Fundamental incompleto & 2 & $13,3 \%$ \\
Fundamental completo & 1 & $6,7 \%$ \\
Ensino médio incompleto & 10 & $66,7 \%$ \\
Ensino médio completo & 2 & $13,3 \%$ \\
\hline
\end{tabular}

\section{Ocupação atual}

\begin{tabular}{lll}
\hline Estudante & 5 & $33,3 \%$ \\
Dona do Lar & 9 & $60 \%$ \\
Trabalho Informal & 1 & $6,7 \%$ \\
\hline
\end{tabular}

Fonte: Dados coletados em Cuité/PB, 2018. Analisados pelo programa SPSS for Windowws versão 22.0.

De acordo com os dados encontrados do perfil socioeconômico, expressos na tabela 1, é importante observar que houve a presença assídua de apenas dois: o grupo de $1 / 2$ até 1 salário mínimo e o grupo de acima de 1 até 2 salários mínimos, considerados como baixa renda. Resultados semelhantes foram encontrados por Moraes, Moraes \& Ribeiro (2014), que ao 
analisarem o perfil epidemiológico de adolescentes grávidas no estado do Pará, encontraram a prevalência de uma renda mensal de até 1 salário mínimo.

Uma baixa renda pode oferecer alguns riscos para aquelas mulheres que estão vivenciando a gestação, uma vez que a instabilidade econômica afeta a saúde da população. Além do fato de que condições sociodemográficas desfavoráveis são vistas como situação de vulnerabilidade. No que condiz à adolescência, a gravidez tende a comprometer, dificultar ou agravar o orçamento familiar (Xavier et al., 2013).

É importante ressaltar que $60 \%$ das adolescentes não exercerem atividade remunerada (alegando serem Donas do Lar), e apenas 6,7\% exerce algum trabalho informal. Além disso, 80\% das entrevistadas afirmaram receber algum tipo de Benefício de Transferência de Renda. Este fato justifica os resultados de baixa renda encontrados neste estudo, trazendo a percepção de dependência, por parte das adolescentes, do salário de terceiros (companheiro e/ou pais) ou com benefícios sociais do governo federal.

Com relação a densidade de morador por cômodo, prevaleceu apenas dois grupos: maior que 0,5 até 1 morador por cômodo (80\%) e maior que 1 até 2 moradores por cômodos (20\%). Os resultados obtidos demonstram que as gestantes adolescentes não residem sozinhas, o que é um fator importante para enfrentar as dificuldades da gravidez. Dados do Censo Demográfico mostram que a população Brasileira, em sua maioria possui até 0,5 morador por cômodo, seguido do grupo que possui mais que 0,5 até 1 morador por cômodo. Segundo este mesmo Censo, esta variável visa analisar a qualidade de vida.

Quando questionadas acerca de sua escolaridade, todas relataram ser alfabetizadas, entretanto apenas 33,3\% estavam frequentando no momento da entrevista. De todas as gestantes, um total de $66,7 \%$, representando a maioria possuíam o Ensino Médio Incompleto como grau de escolaridade.

Diante disso, Vitolo, Gama \& Campagnolo (2010) mencionam que a baixa escolaridade das gestantes reflete diretamente numa menor busca aos serviços de saúde para a assistência pré-natal, o que seria prejudicial.

Além disso, a literatura aponta, também, que a evasão escolar é difícil de ser revertida, uma vez que a adolescente se afasta dos estudos no intuito de cuidar do seu filho tornando a volta à escola mais complicada (Oner et al., 2012).

É possível observar na Tabela 2, o Estado Nutricional (EN) das gestantes de acordo com a renda. 
Tabela 2. Relação entre a Renda Média Familiar e o Estado Nutricional de gestantes adolescentes do município de Cuité/PB, 2018.

\begin{tabular}{l|l|c|c}
\hline \multicolumn{1}{c}{ RENDA MÉDIA FAMILIAR } \\
\hline ESTADO NUTRICIONAL & $\begin{array}{c}\text { Mais de 1/2 a 1 } \\
\text { salário } \\
\text { mínimo* }\end{array}$ & $\begin{array}{c}\text { Mais de 1 a 2 } \\
\text { salários } \\
\text { mínimos }\end{array}$ & Total \\
\hline Eutrofia & $7(46,7 \%)$ & - & 7 \\
Sobrepeso & $3(20 \%)$ & $2(13,3 \%)$ & 5 \\
Obesidade & $2(13,3 \%)$ & $1(6,7 \%)$ & 3 \\
\hline TOTAL & 12 & 3 \\
\hline
\end{tabular}

*Salário base utilizado: R \$954,00 (IBGE, 2018).

Fonte: Dados coletados em Cuité/PB, 2018. Analisados pelo programa SPSS for Windows versão 22.0 .

É interessante analisar e observar na tabela acima o fato de que quase metade das entrevistadas estava eutrófica: 46,7\% e recebiam mais de 1/2 a 1 salário mínimo. Nota-se também que o restante apresentou tanto sobrepeso como obesidade, seja com mais de $1 / 2$ a 1 salário mínimo ou mais de 1 a 2 salários mínimos. Nenhuma gestante encontrou-se com baixo peso.

Visto isso, é importante ressaltar que a ocorrência de alguma inadequação do estado nutricional materno acarreta em inúmeras inconformidades no decorrer da gestação, atingindo, também, o feto. Isto é, gestantes com peso insuficiente ou com sobrepeso e/ou obesidade possuem mais chances de terem filhos com peso inadequado, dentre outras complicações pré e pós-parto (Gonçalves et al., 2012).

Resultados semelhantes foram encontrados por Alves et al. (2016), que ao estudarem o EN de gestantes atendidas em Rondônia, visualizaram a prevalência de sobrepeso e obesidade (47,9\%). Seguindo o mesmo raciocínio, Ferreira \& Benício (2015), revelaram que a maior parte (cerca de 40\%) de mulheres que se apresentavam acima do peso pertencia a classes sociais desfavorecidas.

Um estudo realizado por Hoffman (2012) revela que quanto menor a renda per capita, maior se torna a probabilidade da ocorrência de obesidade e sobrepeso em mulheres. Dessa forma, entende-se que uma renda inferior nem sempre é sinônimo de desnutrição/baixo peso, 
Research, Society and Development, v. 9, n. 7, e169973976, 2020

(CC BY 4.0) | ISSN 2525-3409 | DOI: http://dx.doi.org/10.33448/rsd-v9i7.3976

pois muitas vezes a falta de dinheiro influencia a compra de alimentos de alto valor energético por terem um menor valor de mercado, porém são facilitadores do ganho ponderal e prejudicam a composição corporal, consequentemente comprometendo a saúde e a qualidade de vida.

O ganho de peso excessivo na gestação é preocupante, uma vez que se associa a inúmeras intercorrências, tais como: diabetes gestacional, macrossomia, síndromes hipertensivas da gravidez, dentre outras (ABESO, 2016).

Considerando-se a existência de apenas 2 grupos de renda familiar, os quais já foram discutidos como vulneráveis, estes serão avaliados em conjunto neste ponto, para uma maior veracidade dos resultados. A alimentação é um fator primordial para a manutenção e qualidade da vida.

O consumo inadequado dos alimentos pode acarretar diversos tipos de doenças. Devido a sua importância, boa parte da renda de um domicílio é destinada a este fim. No presente estudo, constatou-se que $88,7 \%$ das entrevistadas destinavam uma quantia de até $\mathrm{R} \$ 500,00$ (quinhentos reais) apenas para alimentação.

Abreu, Hor-Meyll \& Nogueira (2014) explicam que, em famílias de baixa renda, a alimentação é um dos gastos prioritários do orçamento da casa, pois é vista como um item básico.

A partir do QFA foi possível analisar os principais alimentos mais frequentes na alimentação das jovens gestantes, assim como os menos frequentes, agrupados de acordo com o Guia Alimentar para a População Brasileira. Tais alimentos podem ser vistos na Tabela 3. 
Tabela 3. Frequência de consumo dos alimentos das gestantes adolescentes do município de Cuité/PB, 2018.

\begin{tabular}{|c|c|c|c|}
\hline $\begin{array}{l}\text { ALIMENTOS MAIS } \\
\text { FREQUENTES }\end{array}$ & TOTAL & $\begin{array}{c}\text { ALIMENTOS MENOS } \\
\text { FREQUENTES }\end{array}$ & TOTAL \\
\hline \multicolumn{4}{|c|}{ In natura ou Minimamente processados } \\
\hline Frango & $14(93,3 \%)$ & Fígado/miúdos & $12(80 \%)$ \\
\hline Carne Guisada & $13(86,6 \%)$ & Repolho & $12(80 \%)$ \\
\hline Ovo & $13(86,6 \%)$ & Beterraba & $12(80 \%)$ \\
\hline Banana & $14(93,3 \%)$ & Jerimum & $14(93,3 \%)$ \\
\hline Feijão Cozido & $14(93,3 \%)$ & Couve & $15(100 \%)$ \\
\hline Arroz Branco & $12(80 \%)$ & Melancia/melão & $11(73,4 \%)$ \\
\hline Macarrão & $14(93,3 \%)$ & Manga & $12(80 \%)$ \\
\hline Café & $12(80 \%)$ & Castanhas & $15(100 \%)$ \\
\hline \multirow{2}{*}{ Suco de Fruta } & $13(86,6 \%)$ & Arroz Integral & $15(100 \%)$ \\
\hline & & Iogurte Natural & $15(100 \%)$ \\
\hline \multicolumn{4}{|c|}{ Processados } \\
\hline \multirow[t]{4}{*}{ Pão francês/forma } & $14(93,3 \%)$ & Queijo Coalho & $13(86,6 \%)$ \\
\hline & & Pão Integral & $15(100 \%)$ \\
\hline & & Milho Verde & $13(76,6 \%)$ \\
\hline & & Geleia/melado & $15(100 \%)$ \\
\hline \multicolumn{4}{|c|}{ Ultraprocessados } \\
\hline Refrigerante & $12(80 \%)$ & Sorvete & $11(73,4 \%)$ \\
\hline Embutidos & $11(73,3 \%)$ & & \\
\hline Margarina & $14(93,3 \%)$ & & \\
\hline Bolo & $13(86,7 \%)$ & & \\
\hline Bolacha com recheio & $11(73,4 \%)$ & & \\
\hline Bolacha salgada & $14(93,3 \%)$ & & \\
\hline Pipoca chips & $11(73,4 \%)$ & & \\
\hline Chocolate & $11(73,4 \%)$ & & \\
\hline \multicolumn{4}{|c|}{ Óleos, gorduras, sal e açúcar } \\
\hline Açúcar & $15(100 \%)$ & Manteiga & $11(73,4 \%)$ \\
\hline
\end{tabular}

Fonte: Dados coletados em Cuité/PB, 2018. Analisados pelo programa SPSS for Windowws v. 22.0.

Observa-se na Tabela acima os alimentos mais citados nos 4 grupos alimentares. Dentre essa lista, nota-se que as gestantes dão preferência aos alimentos pertencentes ao grupo dos ultraprocessados, que se tratam de alimentos pobres em nutrientes e tendem a ser consumidos com mais frequência, devido a sua composição e apresentação. Esse dado é fundamentado devido o fato de que os alimentos ultraprocessados estão cada vez mais acessíveis no mercado, podendo ser encontrados em sua forma pré-pronta ou pronta para o consumo (Monteiro et al., 2010). 
Moraes e Assunção (2020), ao avaliarem o perfil alimentar de gestantes adolescentes apontam que grande parte do valor energético médio diário, resultando em $37,4 \%$ são oriundos de alimentos industrializados, corroborando com os achados nesse estudo.

Já no grupo dos alimentos menos frequentes, podemos ver a prevalência de alimentos in natura ou minimamente processados, mesmo estando nas recomendações do Guia Alimentar para a População Brasileira que estes devem ser a base da alimentação, pois são alimentos que passaram por pouco ou nenhum processo, além de promover um sistema alimentar sustentável (Brasil, 2014).

Os alimentos in natura ou minimamente processados são basicamente frutas, verduras, legumes, leites in natura, dentre outros, e estes alimentos são responsáveis pela maior parte do aporte nutricional de uma dieta adequada, a qual é de extrema importância na gestação e na adolescência (Araújo, 2015).

A maior parte dos alimentos in natura ou minimamente processados que compõe o grupo de vegetais parece não fazer parte do cotidiano das grávidas, em contrapartida, alimentos com alta densidade calórica e pobres nutricionalmente como refrigerantes, embutidos e salgadinhos eram parte do hábito alimentar das mesmas, representando um risco para a gestação, tendo em vista que o feto depende única e exclusivamente das reservas nutricionais e ingestão materna para se nutrir (Gomes, 2019).

Estudos similares reforçam esses dados, em que alimentos ricos em carboidratos simples, gorduras saturadas e colesterol são escolhas prioritárias na alimentação de gestantes adolescentes, e alimentos ricos em fibras, vitaminas e minerais fazem pouca parte da rotina alimentar das mesmas (Souza et al., 2013; Gill et al., 2015; Moraes, Moraes \& Ribeiro, 2014).

Alguns autores na literatura, como Teixeira\&Moreira (2016) e Júnior et al (2016), mostram dados semelhantes com os resultados obtidos nessa pesquisa, apontando condições econômicas desfavoráveis como um risco de saúde para a população em geral, especialmente para o público materno-infantil, uma vez que se a renda média mensal for ínfima, reflete diretamente no poder de compra de alimentos saudáveis e no acesso de uma alimentação variada e adequada em situações especiais, como é o caso da gestação na adolescência, além de ser um fator de impacto no estado nutricional tanto para o bebê, como para a mãe. Além disso, mães com hábitos alimentares inadequados por razões de baixa renda, tendem a ter filhos com hábitos errôneos também, dando referência a alimentos processados (Sotero, Cabral \& Silva, 2015).

Alimentos como Refrigerante e Café foram elencados como mais frequentes, representando um total de $80 \%$ de gestantes adolescentes que possuem o hábito de consumi- 
los. Sabe-se da problemática envolvendo ambos os alimentos pelo fato de terem em sua composição a cafeína, Rhee et al. (2015), ao realizar uma meta-análise com estudos observacionais, notou que o consumo materno de cafeína pode estar relacionado com o Baixo Peso ao Nascer. Mendes e Moura (2018) em seu estudo com adolescentes grávidas também encontraram uma maior frequência no consumo de refrigerantes, o que se torna algo alarmante nessa fase gestacional, por se tratar de um produto alimentício rico em açúcar, sódio, corantes e conservantes.

No que concerne à qualidade das escolhas dos alimentos, é possível visualizar que alimentos integrais como pão integral e arroz integral não são os preferidos, uma vez que nenhuma grávida (100\%) apresentou o hábito de consumir esse tipo de comida. Em contrapartida, o arroz branco (80\%) e o pão francês $(93,3 \%)$ são consumidos com frequência. Ademais, foi visto que a Manteiga encontra-se nos alimentos menos frequentes $(73,4 \%)$, enquanto a margarina $(93,3 \%)$ é quase uma unanimidade no hábito alimentar das jovens.

O consumo de açúcar é outro fator relevante, o qual houve uma frequência de $100 \%$, resultado semelhante ao encontrado por Moraes, Moraes \& Ribeiro (2014), evidenciando um consumo elevado de açúcar $(48,6 \%)$, bem como os dados achados por Moraes \& Assunção (2020), apresentando uma alta ingestão de açúcar por adolescentes grávidas (53\%). De acordo com o Guia Alimentar Para a População Brasileira (Brasil, 2014) o consumo desse ingrediente deve ser feito de forma moderada, uma vez que seu consumo frequente e em grande quantidade pode acarretar em doenças metabólicas como obesidade, hipertensão arterial sistêmica, diabetes gestacional, além de aumentar também o risco de pré-eclâmpsia e eclampsia (Reis et al., 2010).

Normalmente, um dos fatores que podem influenciar no consumo alimentar, é o acesso ao alimento, que é diretamente influenciado pela renda. Um achado interessante nesse estudo foi o baixo consumo de jerimum $(93,3 \%)$, queijo coalho $(86,6 \%)$ e manga $(80 \%)$, mesmo sendo alimentos regionais, isto é, alimentos com fácil acesso, custo acessível e alto valor nutritivo. Com base nisso, o consumo de alimentos regionais, além de valorizar a produção dos mesmos respeitando a diversidade da região, torna-se uma alternativa para o combate à insegurança alimentar, portanto deve ser estimulado em todas as faixas etárias e grupos populacionais (Martins et al 2012).

Estudos recentes mostram que os hábitos alimentares da população estão mudando cada vez mais, e isso atinge também a valorização dos produtos regionais, uma vez que há o aumento da compra de produtos processados e ultraprocessados e em contrapartida alimentos 
tradicionais como os citados anteriormente tendem a possuir uma menor participação na alimentação brasileira (Moura Souza et al., 2011).

É importante ressaltar que essas futuras mães, geralmente são quem assume a responsabilidade de escolha alimentar dos filhos, bem como a formação de hábitos alimentares da criança (Gomes et al., 2015), sendo essencial o incentivo de uma alimentação adequada desde a gestação, através da assistência pré-natal, para que possa refletir nas próximas gerações.

Quando questionadas acerca do consumo de bebidas alcóolicas, todas as gestantes negaram o hábito, indo de encontro com os achados na literatura, em que apresenta o mesmo resultado que Moraes, Moraes \& Ribeiro (2014) e Pena et al (2017). Tal dado é justificado pelo fato de se tratar de um período que exige maior responsabilidade nesse quesito, uma vez que, de acordo com Rodrigues (2014), o álcool é uma substância teratogênica, o qual pode repercutir de forma negativa no bebê, podendo levar inclusive ao aborto.

Ressalta-se que os trabalhos citados nesse estudo utilizaram uma população diferente e, alguns, também apresentaram objetivo principal distinto do que foi proposto aqui, demonstrando assim algumas limitações que dificultam a generalização dos resultados. É necessário que haja mais estudos nesse segmento, com a mesma análise dos dados e com uma população mais heterogênea.

\section{Considerações Finais}

Situar-se do contexto que envolve a alimentação e suas deficiências e dificuldades durante a fase gestacional de adolescentes, é um fator primordial para uma maior eficácia na assistência nutricional no pré-natal, além de ser importante para traçar estratégias em saúde pública.

A partir dos dados obtidos, foi possível constatar a prevalência de renda de até no máximo 2 salários mínimos entre as gestantes, classificando-as em uma situação de vulnerabilidade econômica, em que a maioria recebe benefício de transferência de renda como forma de complemento, ou como única renda. Assim como a maior presença de gestantes com sobrepeso e obesidade, sendo sua relação com a renda familiar sugestiva de que os fatores econômicos tendem a influenciar de forma negativa o EN de jovens grávidas.

A troca de alimentos in natura ou minimamente processados pelo consumo de alimentos processados e ultraprocessados foi visto como parte da rotina alimentar das 
entrevistadas, o que pode acarretar em problemas futuros, tanto no decorrer da gestação, como em situações pós-parto. Alimentos ricos em calorias e pobre em nutrientes obtiveram uma alta frequência de consumo, e a conexão desse resultado com a situação socioeconômica das gestantes aponta que a renda e o conhecimento influenciam negativamente no poder de compra de alimentos saudáveis.

Aponta-se como estratégia, no intuito de mudança dessa realidade em que se encontram os hábitos alimentares de gestantes adolescentes, a atuação da equipe multidisciplinar, em especial o profissional nutricionista em parceria com órgãos públicos, para que seja possível, em união, encontrar caminhos e meios viáveis, através de estratégias de prevenção e promoção à saúde e com políticas públicas atuantes, para a superação dos desafios vivenciados pelas gestantes acerca da alimentação.

Um fator limitante desse estudo foi o número da amostra estudada, por se tratar de um município pequeno, com, consecutivamente, uma população reduzida. Sugere-se para futuros trabalhos ampliar a amostra populacional e em diferentes regiões do país para uma maior acurácia nos resultados e estratégias mais eficazes para a assistência nutricional no pré-natal de gestantes adolescentes.

\section{Referências}

ABESO. (2016). Diretrizes brasileiras de obesidade. Associação Brasileira para o Estudo da Obesidade e da Síndrome Metabólica (ABESO).

Abreu, L. G., Alvares, L. F. H. M., \& Nogueira, E. M. C. (2015). Consumo de famílias de baixa renda no Rio de Janeiro: um estudo de segmentação baseada no orçamento familiar. Revista ADM. Made, 18(3), 19-39.

Albuquerque, A. P. U. D., Pitangui, A. C. R., Rodrigues, P. M. G. \& Araújo, C. D. (2017). Prevalence of rapid repeat pregnancy and associated factors in adolescents in Caruaru, Pernambuco. Revista Brasileira de Saúde Materno Infantil, 17(2), 347-354.

Alves, K. D. P. S., de Oliveira, L. M. L., Pedersoli, A. G. A., \& Lemke, G. M. M. N. (2016). Estado nutricional e condições socioeconômicas de gestantes atendidas em uma unidade de saúde da família. Revista Saber Científico, 5(1), 61-68. 
Araújo, E. D. S., Santana, J. D. M., Brito, S. M., \& Santos, D. B. D. (2015). Consumo alimentar de gestantes atendidas em Unidades de Saúde. Mundo saúde (Impr.), 28-27.

Atalah, S. (1997). Propuesta de um nuevo estándar de evaluación nutricional em embarazadas. Rev Med Chile, 125, 1429-1436.

Brasil. Ministério da Saúde. (2014). Guia alimentar para a população brasileira. Ministério da Saúde.

Ferreira, C. R. A., \& Ribeiro, G. T. F. (2010). Aspectos Socioeconômicos da Gravidez na Adolescência e as Ações de Saúde, o caso de Anápolis (GO). Vita et Sanitas, 4(1), 73-89.

Ferreira, R. A. B., \& Benicio, M. H. D. A. (2015). Obesidade em mulheres brasileiras: associação com paridade e nível socioeconômico. Revista Panamericana de Salud Pública, 37, 337-342.

Francisquini, A. R., Higarashi, I. H., Serafim, D., \& Bercini, L. O. (2010). Orientações recebidas durante a gestação, parto e pós-parto por um grupo de puérperas. Ciência, Cuidado e Saúde, 9(4), 743-751.

Gill, M., Feliciano, D., Macdiarmid, J., \& Smith, P. (2015). The environmental impact of nutrition transition in three case study countries. Food Security, 7(3), 493-504.

Gomes, C. D. B., Malta, M. B., Martiniano, A. C. D. A., Bonifácio, L. P. D., \& Carvalhaes, M. A. D. B. L. (2015). Práticas alimentares de gestantes e mulheres não grávidas: há diferenças?. Revista Brasileira de Ginecologia e Obstetrícia, 37(7), 325-332.

Gomes, C. D. B., Vasconcelos, L. G., Cintra, R. M. G. C., Dias, L. C. G. D., \& Carvalhaes, M. A. B. L. (2019). Eating habits of pregnant Brazilian women: an integrative review of the literature. Ciencia \& saude coletiva, 24(6), 2293-2306.

Gonçalves, C. V., Mendoza-Sassi, R. A., Cesar, J. A., Castro, N. B. D., \& Bortolomedi, A. P. (2012). Índice de massa corporal e ganho de peso gestacional como fatores preditores de 
complicações e do desfecho da gravidez. Revista Brasileira de Ginecologia e Obstetrícia, 34(7), 304-309.

Hoffmann, R. (2012). Condicionantes do sobrepeso e da obesidade de adultos no Brasil, 2008-2009. Seg Alim Nutr, 19(2), 1-16.

Junior, C. A. M., Malaguti, C., Moreira, L. D. P. D., Faria, N. C., Rezende, W., Rondelli, R. R., ... \& de Cordoba Lanza, F. (2016). Perfil alimentar e sócio-demográfico de gestantes adolescentes da cidade de São Paulo. Revista Extendere, 4(1).

Kempfer, S. S., Fraga, S. M. N., da Silva Hoffman, A. C., Lazzari, D. D., \& Mafra, T. J. (2012). Contracepção na adolescência: uma questão de autocuidado. Revista de Pesquisa Cuidado é Fundamental Online, 4(3), 2702-2711.

Martins, M. C., Veras, J. E. G., Uchoa, J. L., Pinheiro, P. N. D. C., Vieira, N. F. D. C., \& Ximenes, L. B. (2012). Segurança alimentar e uso de alimentos regionais: validação de um álbum seriado. Revista da Escola de Enfermagem da USP, 46(6), 1354-1361.

Mendes B. C., Moura, P. C. (2018). Avaliação do estado nutricional e do consumo alimentar de gestantes adolescentes de um hospital em Curvelo-MG. Revista Brasileira de Ciências da Vida, 6(3).

Monteiro, C. A., Levy, R. B., Claro, R. M., Castro, I. R. R. D., \& Cannon, G. (2010). A new classification of foods based on the extent and purpose of their processing. Cadernos de saude publica, 26(11), 2039-2049.

Moraes, L. P., Moraes, P. M. O., \& Ribeiro, E. C. D. (2014). Perfil epidemiológico e nutricional de adolescentes grávidas internadas em um hospital de referência do estado do Pará. Rev Para Medi, 28(4), 49-56.

Moraes, M. H. S., \& Assunção, M. D. J. S. M. (2020). Socioeconomic and self-care profile of adolescent pregnant women. Research, Society and Development, 9(5), 69953091. 
Moura Souza, A. D., Bezerra, I. N., Cunha, D. B., \& Sichieri, R. (2011). Avaliação dos marcadores de consumo alimentar do VIGITEL (2007-2009). Revista Brasileira de Epidemiologia, 14, 44-52.

Oliboni, C. M., \& Alvarenga, M. D. S. (2015). Atitudes alimentares e para com o ganho de peso e satisfação corporal de gestantes adolescentes. Revista Brasileira de Ginecologia e Obstetrícia, 37(12), 585-592.

Oner, S., Yapici, G., Kurt, A. O., Sasmaz, T., \& Bugdayci, R. (2012). The sociodemographic factors related with the adolescent pregnancy. Asian Pacific journal of reproduction, 1(2), $135-141$.

Pena, J. C. P., de Oliveira Pedersoli, L., Nunes, M. L., dos Santos Freitas, J. M., \& Fernandes, R. A. Q. (2017). Uso de álcool e tabaco na gestação: influência no peso do recém nascido. Revista Saúde-UNG-Ser, 11(1/2), 74-82.

Pereira, A.S. et al. (2018). Metodologia da pesquisa científica. [e-book]. Santa Maria. Ed. UAB/NTE/UFSM. Disponível em: https://repositorio.ufsm.br/bitstream/handle/1/15824/Lic_Computacao_MetodologiaPesquisa-Cientifica.pdf?sequence=1. Acesso em: 27 Abril 2020.

Reis, Z. S. N., Lage, E. M., Teixeira, P. G., Porto, L. B., Guedes, L. R., Oliveira, É. C. L. D., \& Cabral, A. C. V. (2010). Pré-eclâmpsia precoce e tardia: uma classificação mais adequada para o prognóstico materno e perinatal?. Revista Brasileira de Ginecologia e Obstetrícia, 32(12), 584-590.

Rhee, J., Kim, R., Kim, Y., Tam, M., Lai, Y., Keum, N., \& Oldenburg, C. E. (2015). Maternal caffeine consumption during pregnancy and risk of low birth weight: A dose-response metaanalysis of observational studies. PloS one, 10(7).

Rodrigues, L. P. D. S. (2014). Efeitos no feto da ingestão de álcool durante a gravidez (Doctoral dissertation, $[\mathrm{sn}])$. 
Silva, K. S. D., Rozenberg, R., Bonan, C., Chuva, V. C. C., Costa, S. F. D., \& Gomes, M. A.

D. S. M. (2011). Gravidez recorrente na adolescência e vulnerabilidade social no Rio de Janeiro (RJ, Brasil): uma análise de dados do Sistema de Nascidos Vivos. Ciência \& Saúde Coletiva, 16(5), 2485-2493.

Silva, T. D. A., \& Vasconcelos, S. M. L. (2012). Procedimentos metodológicos empregados em questionários de frequência alimentar elaborados no Brasil: uma revisão sistemática. Revista de Nutrição, 25(6), 785-797.

Sotero, A. M., Cabral, P. C., \& Silva, G. A. P. D. (2015). Fatores socioeconômicos, culturais e demográficos maternos associados ao padrão alimentar de lactentes. Revista Paulista de Pediatria, 33(4), 445-452.

Souza, N. A., Queiroz, L. L. C., da Silva Queiroz, R. C. C., Ribeiro, T. S. F., \& Fonseca, M. D. S. S. (2013). Perfil epidemiológico das gestantes atendidas na consulta de pré-natal de uma unidade básica de saúde em São Luís-MA. Revista de Ciências da Saúde, 15(1).

Tavares, L. F., Castro, I. R. R. D., Levy, R. B., Cardoso, L. D. O., \& Claro, R. M. (2014). Padrões alimentares de adolescentes brasileiros: resultados da Pesquisa Nacional de Saúde do Escolar (PeNSE). Cadernos de Saúde Pública, 30, 2679-2690.

Teixeira, V. H., \& Moreira, P. (2016). Maternal food intake and socioeconomic status to tackle childhood malnutrition. Jornal de pediatria, 92(6), 546-548.

Vitolo, M. R. (2014). Nutrição-da gestação ao envelhecimento. Editora Rubio.

Vitolo, M. R., Gama, C. M., \& Campagnolo, P. D. B. (2010). Frequência de utilização do serviço público de puericultura e fatores associados. Jornal de Pediatria, 86(1), 80-84.

Xavier, R. B., Jannotti, C. B., Silva, K. S. D., \& Martins, A. D. C. (2013). Risco reprodutivo e renda familiar: análise do perfil de gestantes. Ciência \& Saúde Coletiva, 18, 1161-1171. 
Research, Society and Development, v. 9, n. 7, e169973976, 2020

(CC BY 4.0) | ISSN 2525-3409 | DOI: http://dx.doi.org/10.33448/rsd-v9i7.3976

Porcentagem de contribuição de cada autor no manuscrito

Marina Lins Mendes Pinto - 17,5\%

Michelly Pires Queiroz - 16,5\%

Ana Beatriz Macêdo Venâncio Santos - 16,5\%

Naryelle da Rocha Silva - 16,5\%

Maria Tereza Lucena Pereira - 16,5\%

Raíra da Silva Campos - 16,5\% 